\title{
Micro-Biopsy Forceps in the Assessment of Peritoneal Carcinomatosis: A Possible New Indication?
}

\author{
Cecilia Binda ${ }^{1}$,Emanuele Dabizzi ${ }^{2}$,Emanuele Sinagra ${ }^{3,4}$, Adele Fornelli ${ }^{5}$, Luca Saragoni ${ }^{6}$, Vincenzo Cennamo ${ }^{2}$, Andrea Anderloni ${ }^{7}$ \\ and Carlo Fabbri'
}

${ }^{1}$ Gastroenterology and Digestive Endoscopy Unit, Forlì - Cesena Hospitals, AUSL Romagna, Forlì-Cesena, ${ }^{2}$ Gastroenterology and Interventional Endoscopy Unit, Ospedale Maggiore "C.A. Pizzardi", AUSL Bologna, Bologna, 'Servizio di Endoscopia Digestiva, Fondazione Istituto G. Giglio di Cefalù, Cefalù (PA), ${ }^{4}$ Section of Nutrition, Istituto Euromediterraneo di Scienze e Tecnologia (IEMEST), Palermo, ${ }^{5}$ Pathology Unit, Ospedale Maggiore "C.A. Pizzardi", AUSL Bologna, Bologna, ${ }^{6}$ Pathology Unit, Morgagni-Pierantoni Hospital, Forlì, AUSL Romagna, Forlì, ${ }^{7}$ Digestive Endoscopy Unit, Humanitas Clinical and Research Center - IRCCS, Rozzano (MI), Italy

Peritoneal carcinomatosis (PC) is defined as a metastatic involvement of the peritoneum by several other primary sites and it is characterized by a marked worsening of prognosis, with limited treatment opportunities. Subsequently, PC should be ruled out before any invasive treatment is administered. A new through-the-needle micro-biopsy forceps (MF) was recently introduced that permits micro-histology cores. In this case series, we evaluated the feasibility of MF in the assessment of PC to complete patient diagnostic work-ups. Five consecutive patients referred for endoscopic ultrasound staging were sampled using MF. Sampling was feasible in all patients with a technical success of $100 \%$. No adverse events were reported in any cases. This technique was feasible and safe with a technical success rate of $100 \%$. It permitted sampling of peritoneal irregularity, obtained high-quality tissue fragments in all cases, and enabled an additional assessment, i.e., immunohistochemical staining. Clin Endosc 2021;54:613-617

Key Words: Carcinomatosis; Endoscopic ultrasonography; Fine needle biopsy; Peritoneum

\section{INTRODUCTION}

Peritoneal carcinomatosis (PC) is defined as a metastatic involvement of the peritoneum by several other primary sites and it is characterized by a marked worsening of prognosis with limited treatment opportunities. ${ }^{1,2}$ Its detection can be challenging since it carries a variable presentation such as minimal thickening of the omental folds as well as solid masses or nodular deposits on the peritoneal surface in association with ascites in half of the cases. ${ }^{3}$ Due to the poor outcomes of

Received: September 2, 2020 Revised: February 11, 2021

Accepted: February 19, 2021

Correspondence: Cecilia Binda

Gastroenterology and Digestive Endoscopy Unit, Forlì - Cesena Hospitals, AUSL Romagna, Via C. Forlanini 34, Forlì-Cesena 47121, Italy

Tel: +39-543-735026, Fax: +39-543-738659, E-mail: cecilia.binda@gmail.com

ORCID: https://orcid.org/0000-0003-1148-9684

cc This is an Open Access article distributed under the terms of the Creative Commons Attribution Non-Commercial License (http://creativecommons.org/ licenses/by-nc/3.0) which permits unrestricted non-commercial use, distribution, and reproduction in any medium, provided the original work is properly cited. these patients, it is mandatory to rule out PC changes before performing any invasive treatment modality. The routine modalities of cross-sectional imaging, such as computed tomography (CT) and magnetic resonance imaging, although very accurate for tumor staging, present low sensitivity in PC detection. ${ }^{3}$ Moreover, serous effusion cytology has high specificity and positive predictive value but modest sensitivity and negative predictive value. ${ }^{4}$ Therefore, in cases of suspected PC, diagnostic laparoscopy is highly recommended because of the possibility of histologic characterization and primary lesion identification.

Endoscopic ultrasound (EUS) is currently considered a safe and effective alternative to laparoscopy for ascites detection and the PC work-up. ${ }^{5}$ Because of its proximity to the peritoneal cavity, EUS demonstrated high resolution and accuracy at detecting hyperechoic lesions surrounded by anechoic ascites and sample capability for the lesions and the liquid. ${ }^{5}$ Recent studies reported progression to a more advanced disease stage in up to $34 \%$ of cases. ${ }^{6}$ A through-the-needle micro-biopsy forceps (MF) (Moray ${ }^{\mathrm{TM}}$; US Endoscopy, Mentor, OH, USA) 
was recently introduced that enables a micro-histological evaluation in addition to standard cytological assessment. The published preliminary experience focused on the usefulness of this novel device on the differential diagnosis of pancreatic cysts, reporting a clinical success rate of $71-83 \%{ }^{7,8}$ Furthermore, this device showed higher sensitivity than cyst fluid analysis in distinguishing mucinous and non-mucinous cysts, improving the diagnostic yield with macroscopically visible specimen. ${ }^{10}$

In this case series, we evaluated the feasibility of MF in the diagnostic assessment of PC for completing the diagnostic work-up.

\section{CASE REPORT}

We enrolled five consecutive patients with clinically suspicious malignancy and uncertain PC status at the time of the CT scan who underwent EUS staging and tissue sampling in our center. The mean age was 74 years. CT revealed the presence of a primitive tumor in three cases and PC only in two cases. The patients' clinical characteristics are outlined in Table 1.

All procedures were performed by expert endosonogra- phers using a therapeutic linear echoendoscope under the cover of periprocedural antibiotics. After a comprehensive EUS evaluation, ascitic fluid was collected using a 19G fine-needle aspiration (FNA) needle (Expect ${ }^{\mathrm{TM}}$ Slimline; Boston Scientific, Marlborough, MA, USA) passed through the transgastric or transduodenal route until the tip was within the ascites. Before liquid suction, the MF was inserted through-the-needle and the suspected carcinomatosis nodule/peritoneal thickness was sampled (Figs 1,2). This route should avoid any other diseased tissue or organ (e.g., diseased area in gastric cancer) to avoid contamination or misdiagnosis. ${ }^{10} \mathrm{MF}$ has a sheath diameter of $0.8 \mathrm{~mm}$ and a jaw opening of $4.3 \mathrm{~mm}$ (Fig. 3). Micro-histology specimens were collected in formalin, stained with standard hematoxylin and eosin (Fig. 4) and evaluated by dedicated pathologists. Supplementary immunohistochemical staining was also used when needed. Ascitic fluid was collected for cytological analysis.

Care was taken to avoid any intervening vessels or area involved by malignancy to avoid contamination of the sample and peritoneal seeding. ${ }^{11,12}$ Patient demographics, EUS findings, technical success, ascitic fluid analysis, MF sample quality, histopathological results, and adverse events are shown in Table 2.

Table 1. Patients' Clinical Characteristics

\begin{tabular}{cccccc}
\hline Case & Sex & Age $(\mathbf{y r})$ & Evidence of neoplasia at CT scan & PC at CT scan & Ascitis at CT scan \\
\hline 1 & M & 75 & Pancreatic mass & No & No \\
2 & F & 64 & Ovarian mass & Yes & Yes \\
3 & F & 77 & Pancreatic mass & Yes & Yes \\
4 & F & 64 & No & Yes & No \\
5 & M & 89 & Gastric wall thickening & & Yes \\
\hline
\end{tabular}

CT, computed tomography; PC, peritoneal carcinomatosis.

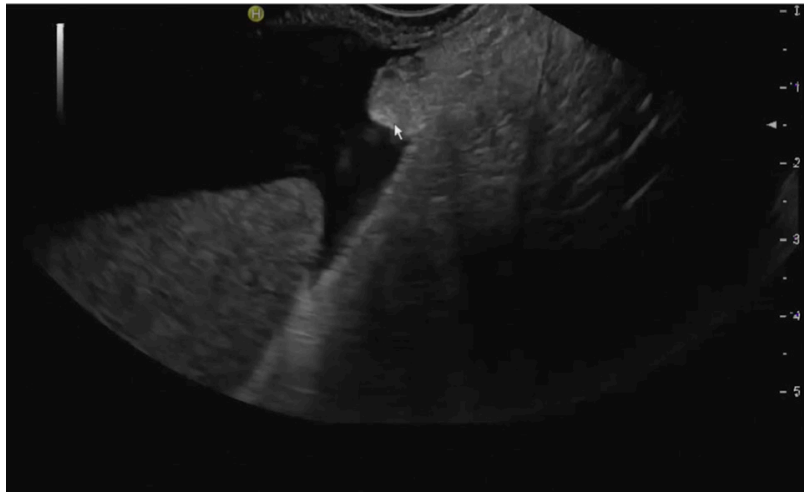

Fig. 1. Endoscopic ultrasound evaluation of a suspicious peritoneal thickening.

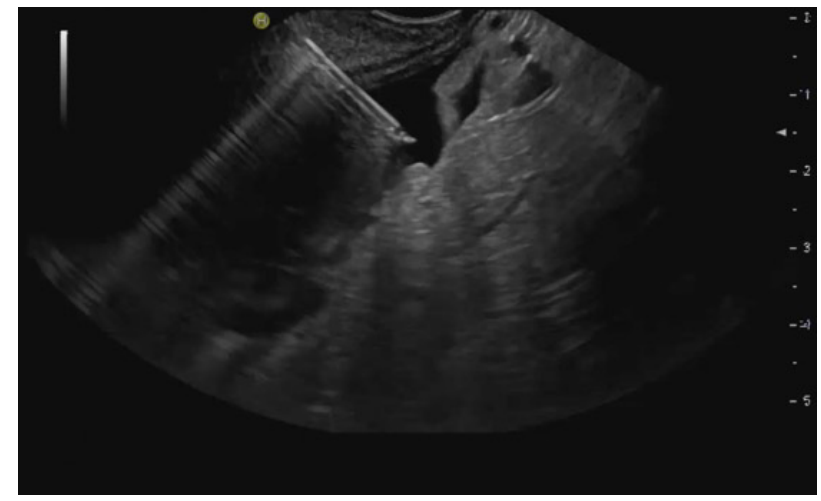

Fig. 2. Endoscopic ultrasound view of Moray ${ }^{\mathrm{TM}}$ (US Endoscopy, Mentor, $\mathrm{OH}$, USA) forceps introduced through a $19 \mathrm{G}$ needle for tissue sampling of a suspected peritoneal nodule. 


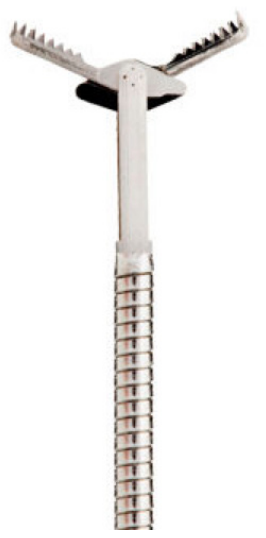

Fig. 3. Moray ${ }^{\mathrm{TM}}$ (US Endoscopy, Mentor, OH, USA) forceps.

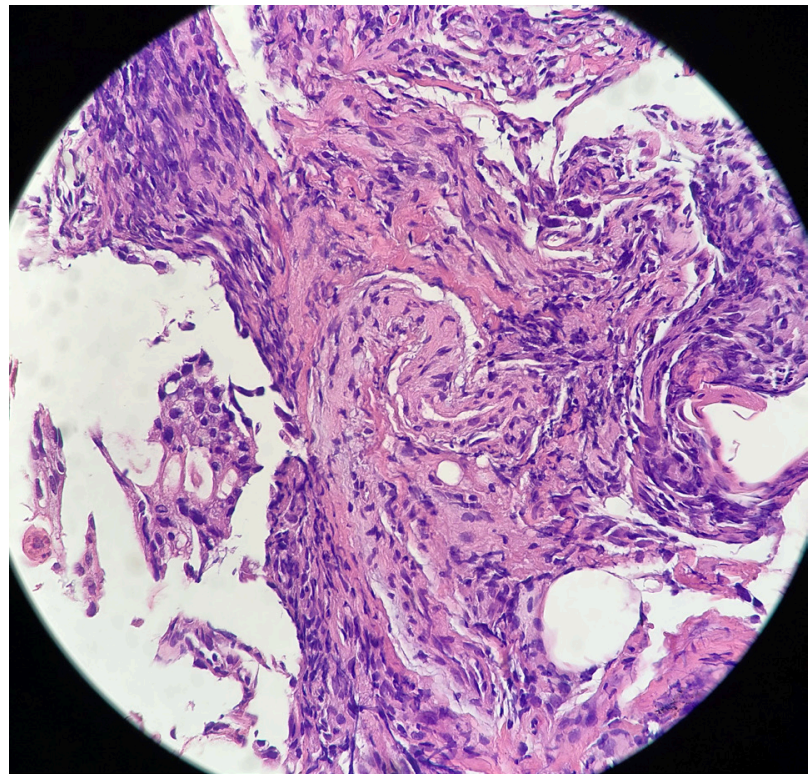

Fig. 4. Hematoxylin and eosin staining (original magnification $\times 400$ ) of a nodule of peritoneal carcinomatosis sampled using of Moray ${ }^{\mathrm{TM}}$ (US Endoscopy, Mentor, OH, USA) forceps. The specimen consists of a small tissue fragment covered by pleomorphic cells with a glandular arrangement. The final diagnosis was adenocarcinoma of gastric origin.
In all patients, EUS revealed the presence of ascites with suspicious peritoneal nodules that were sampled with MF. Ascitic fluid was also collected for cytological analysis.

Patients 1 and 3 also showed a pancreatic mass that was subjected to EUS-guided fine-needle biopsy (FNB) with a subsequent histological diagnosis of pancreatic ductal adenocarcinoma. Patient 2 had a left ovarian mass that was highly suggestive of malignancy on the CT scan. Patient 4 had a history of breast carcinoma; in patient 5, a CT scan showed thickening of the gastric wall, suggestive of neoplasia, which was sampled during EUS procedure and subsequently confirmed histologically.

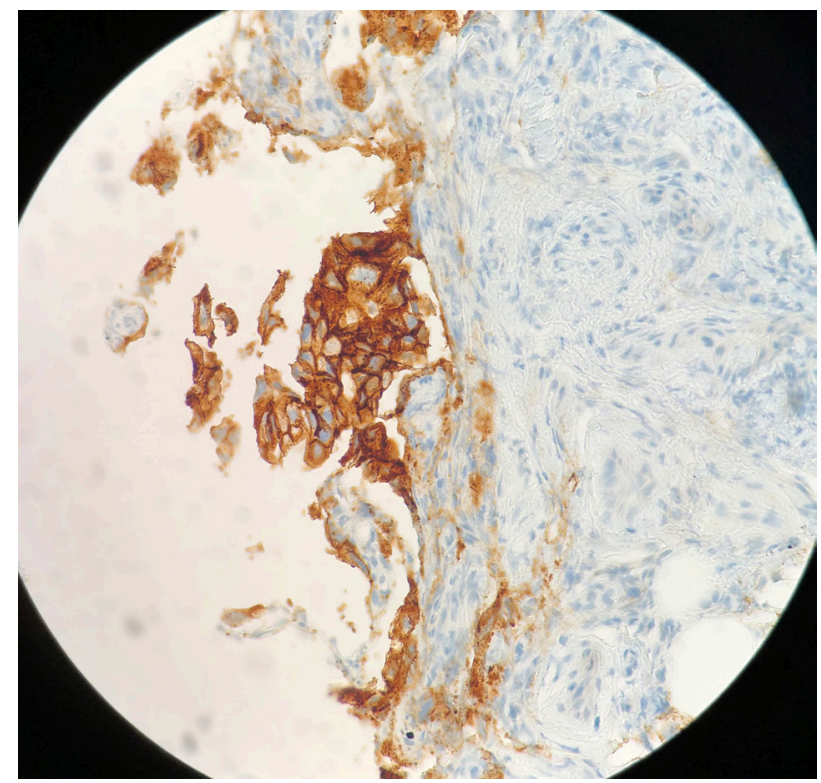

Fig. 5. Ber-EP4 nicely identifies malignant epithelial cells, allowing their distinction from reactive mesothelium (original magnification $\times 400$ ).

Table 2. Results of Pertitoneal Carcinomatosis Sampling by Micro-Biopsy Forceps

\begin{tabular}{cccccc} 
Case & $\begin{array}{c}\text { Histological diagnosis of the } \\
\text { primary tumor }\end{array}$ & Moray on PC & Diagnosis at MF & $\begin{array}{c}\text { Immunohistochemical } \\
\text { stains at MF }\end{array}$ & $\begin{array}{c}\text { Adverse } \\
\text { events }\end{array}$ \\
\hline 1 & PDAC & Diagnostic & Pancreatic adenocarcinoma & Ber-EP4 & None \\
2 & - & Diagnostic & Ovarian adenocarcinoma & Ber-EP4, PAX8 & None \\
3 & PDAC & Diagnostic & Pancreatic adenocarcinoma & Ber-EP4 & None \\
4 & Lobular carcinoma of the breast & Diagnostic & Breast cancer & Ber-EP4, GATA3 & None \\
5 & Gastric adenocarcinoma & Diagnostic & Gastric adenocarcinoma & Ber-EP4 & None \\
\hline
\end{tabular}

PC, peritoneal carcinomatosis; MF, Moray ${ }^{\mathrm{TM}}$ (US Endoscopy, Mentor, OH, USA) Forceps; PDAC, pancreatic ductal adenocarcinoma. 
The technique using this through-the-needle device was feasible and safe with a technical success rate of $100 \%$. In fact, in every case, it enabled the collection of small tissue fragments suitable for histological and immunohistochemical evaluation (Figs 4 and 5).

All MF biopsies were diagnostic of PC: the epithelial nature of the malignant cells was confirmed with Ber-EP4 antibody and, in two cases (patient 2 and patient 4), additional immunohistochemical stains, PAX8 and GATA3, confirmed the clinically suspicious ovarian and breast origins, respectively.

Ascitic fluid cytology revealed the presence of malignant cells in all cases, but given the presence of diagnostic MF biopsies, immunohistochemistry was not performed on the cytological material. In fact, immunohistochemical stains are preferentially performed on histological material because of better technical results and because serial sections permit a more precise evaluation of results.

No adverse events were reported during or after the procedure in any case.

\section{DISCUSSION}

PC is a critical condition with variable presentations characterized by poor prognosis and limited treatment options. Despite the use of different diagnostic modalities, including cross-sectional imaging techniques, explorative diagnostic laparoscopy, and EUS, it may still be misdiagnosed. This may lead to the wrong therapeutic pathway, such as resection, without the finality of radical intervention (R0). A prompt and correct PC characterization is mandatory for patients with neoplastic diseases. The recent availability of a through-the-needle MF overcomes the pitfalls in cytology sampling in several disease work-ups, thus improving the EUS diagnostic yield. Its use has been largely evaluated in the setting of pancreatic cystic lesions, with two recent meta-analyses confirming that throughthe-needle biopsy (TTNB) using MF represents a feasible technique with a high rate of obtaining high-quality specimens for histological assessment. ${ }^{13,14}$ The first study of 490 patients demonstrated a pooled diagnostic accuracy rate, sensitivity and specificity of TTNB of $78.8 \%, 82.2 \%$, and $96.8 \%$, respectively, with a mean of three passes needed to obtain adequate histological samples. ${ }^{13}$ In the second meta-analysis of 460 patients, the technical success rate was $98.5 \%$, histological accuracy rate was $86.7 \%$, and adverse event rate was $8.6 \%{ }^{14}$

Moreover, a study conducted by Crinò et al. ${ }^{15}$ among 61 patients demonstrated that two visible samples reached 100\% histologic adequacy to allow differentiation between mucinous and non-mucinous cysts to obtain a cyst-lining epithe- lium, define the grade of dysplasia, and diagnose the specific histotype of the cysts. Nevertheless, the diagnostic reliability of TTNB sampling versus surgical histology was $90 \%$.

Moreover, the inter-observer agreement among pathologists seems to be very close to perfect, especially regarding the specimen adequacy and the grading of epithelial dysplasia. ${ }^{16}$

Finally, gastric cancer and pancreatic cancer with PC are most likely accompanied by lymph node metastasis; therefore, it is more likely that EUS-FNA/FNB from the lymph nodes or the primary nodule will be better for sampling. However, in our series, we would like to highlight that 1 ) the performance of EUS-FNA depends on many factors, including the location, size, and characteristics of the target lesion, that could make the sampling of peritoneal nodules easier; 2) despite the high sensitivity (94\%) and specificity (98\%) of nodal sampling, as shown in a recent meta-analysis, ${ }^{17}$ peritoneal sampling may be a reliable and safe alternative to lymph node sampling in cases of PC of unknown origin; and 3) the certainty of the diagnosis of a primary tumor in the presence of PC obtained through peritoneal sampling represents a further instrument to complete the staging of the primary tumor and define its prognosis, thus defining the biological aggressiveness of the tumor itself.

To our knowledge, only one case report using MF for the assessment of peritoneal nodules has been reported to date, although in that case, a diagnosis of primary peritoneal serous carcinoma was made. ${ }^{18}$ In our case series, the technique using this through-the-needle device was feasible with a technical success rate of $100 \%$, as well as it was considered safe since no adverse events occurred. It permitted sampling of the peritoneal irregularity, the obtaining of high-quality tissue fragments in all cases, and enabling additional assessments such as immunohistochemical staining. Furthermore, this device allowed us to clarify the diagnosis of the neoplastic disease, improving the staging work-up in patients with PC not seen at other cross-sectional imaging modalities and characterizing the primary tumor in patients with ascites of unknown origin. Moreover, as serous fluid cytology has modest sensitivity, ${ }^{4} \mathrm{MF}$ biopsies of peritoneal nodules or irregularities can be a valuable adjunctive tool to enable a more precise diagnosis.

In conclusion, EUS represents an excellent tool for the detection of small amounts of ascites, while EUS paracentesis may help in the diagnosis of PC. TTNB from peritoneal nodules using MF seems to have a good yield for the diagnosis of PC, but other causes of peritoneal nodules such as tuberculosis, pseudomyxoma, or lymphoma may also be identified. ${ }^{5,6,11,19,20}$ However, further prospective studies are needed with a comparative analysis versus other well-known diagnostic techniques to clarify if EUS with TTNB using MF, 
in conjunction with ascitic fluid cytology, may become a standard tool in the diagnosis of PC.

Conflicts of Interest
Vincenzo Cennamo is consultant for Olympus, Euromedical, Novità
Medicali
Andrea Anderloni is consultant for Boston Scientific and Olympus.
The other authors have no potential conflicts of interest.
Funding
$\begin{array}{ll}\text { None. } & \\ \text { ORCID } & \text { https://orcid.org/0000-0003-1148-9684 } \\ \text { Cecilia Binda: } & \text { https://orcid.org/0000-0001-7916-358X } \\ \text { Emanuele Dabizzi: } & \text { https://orcid.org/0000-0002-8528-0384 } \\ \text { Emanuele Sinagra: } & \text { https://orcid.org/0000-0002-4329-0859 } \\ \text { Adele Fornelli: } & \text { https://orcid.org/0000-0002-2137-9596 } \\ \text { Luca Saragoni: } & \text { https://orcid.org/0000-0001-6733-9226 } \\ \text { Vincenzo Cennamo: } & \text { https://orcid.org/0000-0002-1021-0031 } \\ \text { Andrea Anderloni: } & \text { https://orcid.org/0000-0003-0670-9910 } \\ \text { Carlo Fabbri: } & \end{array}$

\section{REFERENCES}

1. Terzi C, Arslan NC, Canda AE. Peritoneal carcinomatosis of gastrointestinal tumors: where are we now? World J Gastroenterol 2014;20:1437114380.

2. Lemmens VE, Klaver YL, Verwaal VJ, Rutten HJ, Coebergh JWW, de Hingh IH. Predictors and survival of synchronous peritoneal carcinomatosis of colorectal origin: a population-based study. Int J Cancer 2011;128:2717-2725.

3. Patel CM, Sahdev A, Reznek RH. CT, MRI and PET imaging in peritoneal malignancy. Cancer Imaging 2011;11:123-139.

4. Lobo C, Costa J, Petronilho S, Monteiro P, Leça L, Schmitt F. Cytohistological correlation in serous effusions using the newly proposed international system for reporting serous fluid cytopathology: experience of an oncological center. Diagn Cytopathol 2021;49:596-605.

5. Rana SS, Bhasin DK, Srinivasan R, Singh K. Endoscopic ultrasound-guided fine needle aspiration of peritoneal nodules in patients with ascites of unknown cause. Endoscopy 2011;43:1010-1013.

6. Levy MJ, Abu Dayyeh BK, Fujii LL, et al. Detection of peritoneal carcinomatosis by EUS fine-needle aspiration: impact on staging and resectability (with videos). Gastrointest Endosc 2015;81:1215-1224.
7. Kovacevic B, Karstensen JG, Havre RF, et al. Initial experience with EUS-guided microbiopsy forceps in diagnosing pancreatic cystic lesions: a multicenter feasibility study (with video). Endosc Ultrasound 2018;7:383-388.

8. Barresi L, Crinò SF, Fabbri C, et al. Endoscopic ultrasound-through-the-needle biopsy in pancreatic cystic lesions: A multicenter study. Dig Endosc 2018;30:760-770.

9. Zhang ML, Arpin RN, Brugge WR, Forcione DG, Basar O, Pitman MB. Moray micro forceps biopsy improves the diagnosis of specific pancreatic cysts. Cancer Cytopathol 2018;126:414-420.

10. Crinò SF, Bernardoni L, Brozzi L, et al. Association between macroscopically visible tissue samples and diagnostic accuracy of EUS-guided through-the-needle microforceps biopsy sampling of pancreatic cystic lesions. Gastrointest Endosc 2019;90:933-943.

11. Sharma V, Rana SS, Ahmed SU, Guleria S, Sharma R, Gupta R. Endoscopic ultrasound-guided fine-needle aspiration from ascites and peritoneal nodules: a scoping review. Endosc Ultrasound 2017;6:382-388.

12. Nguyen PT, Chang KJ. EUS in the detection of ascites and EUS-guided paracentesis. Gastrointest Endosc 2001;54:336-339.

13. Facciorusso A, Del Prete V, Antonino M, Buccino VR, Wani S. Diagnostic yield of EUS-guided through-the-needle biopsy in pancreatic cysts: a meta-analysis. Gastrointest Endosc 2020;92:1-8.e3.

14. Tacelli M, Celsa C, Magro B, et al. Diagnostic performance of endoscopic ultrasound through-the-needle microforceps biopsy of pancreatic cystic lesions: systematic review with meta-analysis. Dig Endosc 2020;32:1018-1030.

15. Crinò SF, Bernardoni L, Brozzi L, et al. Association between macroscopically visible tissue samples and diagnostic accuracy of EUS-guided through-the-needle microforceps biopsy sampling of pancreatic cystic lesions. Gastrointest Endosc 2019;90:933-943.

16. Larghi A, Manfrin E, Fabbri C, et al. Interobserver agreement among expert pathologists on through-the-needle microforceps biopsy samples for evaluation of pancreatic cystic lesions. Gastrointest Endosc 2019;90:784-792.e4.

17. Li C, Shuai Y, Zhou X. Endoscopic ultrasound guided fine needle aspiration for the diagnosis of intra-abdominal lymphadenopathy: a systematic review and meta-analysis. Scand J Gastroenterol 2020;55:114-122.

18. Saito K, Nakai Y, Ushiku T, et al. Gastrointestinal: successful diagnosis of primary peritoneal serous carcinoma by endoscopic ultrasound-guided through-the-needle forceps biopsy. J Gastroenterol Hepatol 2019; 34:1271.

19. Kocaman O, Danalioğlu A, İnce AT, Tozlu M, Şentürk H. Diagnosis of tuberculous peritonitis using endoscopic ultrasound-guided fine-needle aspiration biopsy of the peritoneum. Turk J Gastroenterol 2013;24:6569.

20. Peter S, Eltoum I, Eloubeidi MA. EUS-guided FNA of peritoneal carcinomatosis in patients with unknown primary malignancy. Gastrointest Endosc 2009;70:1266-1270. 